\title{
FACT - Multi-wavelength analysis of more than 30 flares of Mrk 421
}

\author{
V. Sliusar ${ }^{1}$, A. Arbet-Engels ${ }^{2}$, D. Baack ${ }^{3}$, M. Balbo ${ }^{1}$, M. Beck ${ }^{2, a}$, A. Biland ${ }^{2}$, M. Blank ${ }^{4}$, \\ T. Bretz ${ }^{2, a}$, K. Bruegge ${ }^{3}$, M. Bulinski ${ }^{3}$, J. Buss ${ }^{3}$, M. Doerr ${ }^{4}$, D. Dorner ${ }^{4}$, D. Elsaesser ${ }^{3}$, \\ D. Hildebrand ${ }^{2}$, R. lotov ${ }^{4}$, M. Klinger ${ }^{2, a}$, K. Mannheim ${ }^{4}$, S.A. Mueller ${ }^{2}$, D. Neise ${ }^{2}$, \\ M. Noethe ${ }^{3}$, A. Paravac ${ }^{4}$, W. Rhode ${ }^{3}$, B. Schleicher ${ }^{4}$, K. Sedlaczek ${ }^{3}$, A. Shukla ${ }^{4}$, L. Tani ${ }^{2}$, \\ F. Theissen ${ }^{2, a}$, R. Walter* ${ }^{* 1}$ and E. von Willert ${ }^{4}$ \\ (the FACT Collaboration ${ }^{\dagger}$ ) \\ ${ }^{1}$ University of Geneva, Department of Astronomy, Chemin d'Ecogia 16, 1290 Versoix, \\ Switzerland \\ ${ }^{2}$ ETH Zurich, Institute for Particle Physics and Astrophysics, Otto-Stern-Weg 5, 8093 Zurich, \\ Switzerland \\ ${ }^{3}$ TU Dortmund, Experimental Physics 5, Otto-Hahn-Str. 4, 44221 Dortmund, Germany \\ ${ }^{4}$ Universität Würzburg, Institute for Theoretical Physics and Astrophysics, Emil-Fischer-Str. 31, \\ 97074 Würzburg, Germany \\ ${ }^{a}$ also at RWTH Aachen University, Physics Institute III A, 52074 Aachen, Germany \\ E-mails: vitalii.sliusar@inige.ch, roland.walter@unige.ch
}

\begin{abstract}
Mrk 421 is a high-synchrotron-peaked blazar featuring bright and persistent $\mathrm{GeV}$ and $\mathrm{TeV}$ emission. We use multi-wavelength light curves of Mrk 421 spanning 5.5 years with FACT (TeV) and Fermi LAT $(\mathrm{GeV})$ in the gamma rays, Swift BAT, Swift XRT and MAXI in the X-rays, together with optical and radio data and investigate the physical processes driving the emission and variability. Observations by FACT are continuous and not triggered, so the source was found in a wide range of flux states and more than 30 flares were identified from X-rays to TeV. The light curves in $\mathrm{TeV}$ and $\mathrm{X}$-rays feature very similar flares with rise and decay times of a few days and zero lag, characteristic for electron processes. At least two parameters per flare, the amplitude and the cut-off energy, are required to explain the observed variability. In addition, the GeV light curve leads and is strongly correlated with the optical and radio light curves as expected from SSC emitting shock propagating in a conical jet.
\end{abstract}

36th International Cosmic Ray Conference -ICRC2019-

July 24th - August 1st, 2019

Madison, WI, U.S.A.

\footnotetext{
*Speaker.

${ }^{\dagger}$ for collaboration list see PoS(ICRC2019)1177
} 


\section{Introduction}

Mrk 421 is one of the brightest and closest high-energy-peaked blazars $(z=0.031)$. It features a bright and persistent $\mathrm{GeV}$ and $\mathrm{TeV}$ emission with frequent flaring activities. Its average spectral energy distribution has been fitted with different models: e.g.: one zone leptonic synchrotron self-Compton model (SSC) [1], hadronic model where the accelerated protons cool through synchrotron emission [2] or interact with the leptonic synchrotron photons to create cascade of pions and muons, decaying in $\gamma$-rays and neutrinos [3]. Fast variations suggest, that either a shock moves relativistically within the jet or the emission region is much smaller than the gravitational radius [4], and is driven by the interactions between stars/clouds and the jet or by magnetic reconnections.

To the date, Mrk 421 was a target of multiple multi-wavelength campaigns [e.g. 5, 6]. During the 2009 campaign, Mrk 421 was simultaneously observed from the radio to the $\mathrm{TeV}$ band for 4.5 months in the absence of a strong flare [6]. The fractional variability revealed that most variability lies in the X-ray $\left(F_{v a r}=0.5\right)$ and $\mathrm{TeV}\left(F_{v a r}=0.3\right)$ bands. A harder-when-brighter behaviour was discovered in the $\mathrm{X}$-rays. The smallest variations were observed in the radio. A positive cross-correlation was found between $\mathrm{X}$-rays and $\mathrm{TeVs}$ at a maximum lag of $\sim 5$ days. No strong correlations between optical/UV and X-rays were found.

In this paper, we report results from a MWL analysis using 5.5 years of the FACT monitoring in the $\mathrm{TeV}$ band, which is ongoing for more than 7 years [7]. The observations were not triggered, therefore unbiased, and as regular as possible taking into account observing conditions constraints. We also used continuous radio, optical, ultraviolet, $\mathrm{X}$-ray, $\mathrm{GeV}$ and $\mathrm{TeV}$ light curves obtained quasi-simultaneously with FACT with the aim to investigate various emission models.

\section{Multi-wavelength data}

Data from nine different instruments was used to build the light curves spanning between December 14, 2012 and April 18, 2018. We performed the cross-correlation, auto-correlation, Bayesian Block and fractional variability analysis using this dataset to investigate the physical processes responsible for the emission in all bands. During the considered period, Mrk 421 was observed at various flux states in all bands. Flares observed from the $\mathrm{X}$-rays to the $\mathrm{TeV}$ are narrow enough to be identified individually (see table 1). At longer wavelength the flares become wider and eventually overlap.

The $\mathrm{TeV}$ data were obtained by the First G-APD Cherenkov Telescope (FACT), which is a $3.8 \mathrm{~m}$ imaging air Cherenkov telescope located at La Palma [8]. Since the start of data taking in 2011, the system gradually was automatized, resulting in remote operation since late July 2012 and fully robotic operation since 2017. This allows to perform long-term regular observations of bright $\mathrm{TeV}$ sources with high cadence. Due to the use of a SiPM camera and a state-of-the-art feedback system, the telescope can operate in bright ambient light conditions [7]. A detailed description of events reconstruction and analysis is presented in [9]. The quality checks and background suppression techniques are described in [10], [11] and [12]. The energy threshold based on simulated data is determined to be $775 \mathrm{GeV}$ for sources with Crab-Nebula-like spectrum and $\sim 860 \mathrm{GeV}$ for Mrk 421 (harder spectrum). 
The Mrk 421 data in $\mathrm{GeV} \gamma$-rays with energy $100 \mathrm{MeV}<\mathrm{E}<300 \mathrm{GeV}$ was obtained by the Fermi Large Area Telescope (LAT)[13]. The PASS8 pipeline and Fermi Science Tool v10r0p5 package were used to process the data. The fitting model included sources from the LAT 4-year Point Source Catalogue.

X-ray observations in the $15-50 \mathrm{keV}$ band were performed by Swift/BAT. The reduction pipeline is based on the BAT analysis software HEASOFT version 6.13 [14]. The daily light curve was built using observational data from 29344 orbital periods or almost 5.5 years.

X-ray observations in the $0.2-10 \mathrm{keV}$ band were performed by the Swift/XRT X-ray telescope [15]. The light curve was obtained from the on-line Swift-XRT products generation tool ${ }^{1}$, which uses HEASOFT software version 6.22 .

X-ray observation from 2 to $20 \mathrm{keV}$ were performed by MAXI instrument [16]. The light curve for Mrk 421 from MAXI is publicly available ${ }^{2}$.

The UV observations were performed by Swift/UVOT telescope in three bands UVW1, UVM2 and UVW2 [17]. The data were reduced using on-, off-method using the HEASOFT package version 6.24 along with UVOT CALDB version 20170922.

The V-band data from $1.54 \mathrm{~m}$ Kuiper Telescope on Mountain Bigelow and the $2.3 \mathrm{~m}$ Bok Telescope on Kitt Peak were used for the monitoring [18]. Our dataset includes data from Cycle 5 to 10 . The light curves are available publicly ${ }^{3}$.

Mrk 421 observations are regularly performed in the radio by the OVRO 40 meter radio telescope. Observations were performed twice per week at $15 \mathrm{GHz}$. The data are available from the telescope archive publicly ${ }^{4}$.

\section{Light curves timing and correlation analysis}

Using multi-wavelength light curves, we calculated values of fractional variability, lags and correlations between different bands, found best-fit parameters of the $\mathrm{GeV}$ to radio response profile.

\subsection{Fractional variability}

We performed the fractional variability analysis of all the light curves of Mrk 421 as proposed in [19]. The uncertainties were estimated following the [20]. Using the 5.5 years of multiwavelength observations, we found that the lowest $F_{\text {var }}=0.15$ is in radio and the highest $\sim 1.33$ in X-rays (Swift/BAT). Then the fractional variability drops to $\sim 0.34$ for Fermi LAT and increases to $\sim 0.92$ in TeV making two distinctive humps in the $F_{\text {var }}$ dependency of the frequency. Previous studies of the fractional variability were reported in [6] and [21], while having the same two hump behaviour, the amplitude was three time lower, which likely can be explained by much shorter light curves being used.

Two maxima of the fractional variability of Mrk 421 indicate that the high energy portions of the two emission components are more variable than the low energy ones. This suggests that the cutoff energies of both components are the primary source of the variability. Similarly, we verified

\footnotetext{
${ }^{1}$ http://www.swift.ac.uk/user_objects/

${ }^{2}$ http://maxi.riken.jp/star_data/J1104+382/J1104+382.html

${ }^{3}$ http://james.as.arizona.edu/ psmith/Fermi/DATA/photdata.html

${ }^{4} \mathrm{http}: / /$ www.astro.caltech.edu/ovroblazars/
} 
that the simultaneous flux for the $\mathrm{TeV}$ and $\mathrm{X}$-ray is well linked, underlying that a single parameter is driving these variations.

\subsection{Correlations}

To investigate the connection between the emission in different bands, we calculate the crosscorrelation between all the light curves. We use DCF [22] to estimate the correlation between irregularly sampled data. We adopted 1 day bins for most DCFs due to long light curves being used (except when the data were more sparse). Uncertainties in DCF are in most cased underestimated, so we adopted Monte Carlo simulations to calculate the lag probability. The lag uncertainty corresponds to the standard deviation of the distribution of the lags obtained for the random subsets.

To estimate the variability timescales, we calculate the discrete auto-correlation functions from radio to $\mathrm{TeV}$. The uncertainties are estimated using Monte Carlo simulations similarly to the case of DCFs. We find that variability time scale observed in the $\mathrm{TeV}$ and $\mathrm{X}$-ray bands are short, $\sim 3$ days, which is consistent with the models where emission is due to fast relativistic electron cooling. The same quantity for the GeV Fermi LAT light curve is even smaller ( $\lesssim 1$ day), which might be an evidence of different parameters driving the variability in that band.

We report a strong correlation between $\mathrm{TeV}$ and X-ray light curves (Swift/XRT, Swift/BAT, MAXI) (Fig. 1). We found no significant lag between $\mathrm{TeV}$ and $\mathrm{X}$-rays, the combined lag is $(0.26 \pm 0.46)$ days $(1 \sigma)$. This result is consistent with previously reported lags on more sparse datasets $[6,23]$. The cross-correlation between $\mathrm{GeV}$ and $\mathrm{TeV}$ or $\mathrm{GeV}$ and $\mathrm{X}$-rays is weak and the lag cannot be identified reliably.
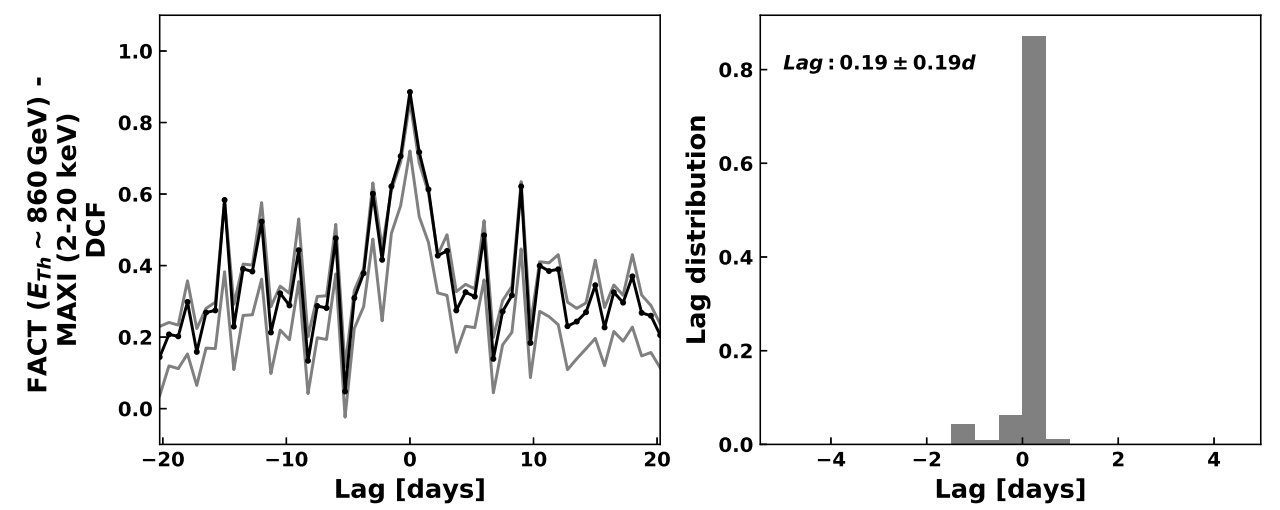

Figure 1: DCF cross-correlations of TeV (FACT) and X-rays (MAXI) light curves. One day binning was used. Left: DCF values as a function of lag. Gray lines denote the $1 \sigma$ uncertainties. Right: lag distribution corresponding to the maximum DCF value.

To identify individual flares in X-rays, $\mathrm{GeV}$ and $\mathrm{TeV}$ bands, we applied the Bayesian Block algorithm [24] to each light curve and compared the times of the flares, which in this case we define as statistically significant block with amplitude two sigma above the previous block and duration of at least 2 days. The false positive probability was set to 5\% [24]. All flares based on the Fermi LAT, Swift/XRT, MAXI and FACT light curves are listed in table 1.18 of the TeV flares were detected in the $\mathrm{GeV}$ and $\mathrm{X}$-rays, but 4 flares in $\mathrm{GeV}$ band were not detected in any other highenergy band. This indicates that particle populations with different spectra are necessary to explain all observations. 
Table 1: List of gamma ray and X-ray flares sorted by the spectral bands in which they are detected.

\begin{tabular}{|c|c|c|}
\hline Bands & Number & Time ranges, MJD \\
\hline TeV only: & 2 & 56689-56692, 57006-57015 \\
\hline GeV only: & 4 & $\begin{array}{l}56275-56284,56291-56296,56368-56376, \\
57064-57073\end{array}$ \\
\hline $\mathrm{TeV}$ and $\mathrm{X}$-rays: & 11 & $\begin{array}{lll}56441-56449, & 56696-56700, & 57110-57121, \\
57368-57379, & 57385-57390, & 57422-57431, \\
57531-57538, & 57728-57753, & 57770-57775, \\
57787-57793, & 58103-58113 & \end{array}$ \\
\hline TeV, GeV, X-rays: & 18 & $\begin{array}{lll}56317-56330, & 56369-56383, & 56389-56400, \\
56650-56670, & 56751-56755, & 56976-57005, \\
57039-57053, & 57065-57070, & 57091-57099, \\
57188-57192, & 57432-57449, & 57504-57511, \\
57545-57550, & 57756-57769, & 57850-57866, \\
58129-58142, & 58162-58167, & 58185-58196\end{array}$ \\
\hline
\end{tabular}

The V-band and UV light curves are highly correlated with near to zero lag despite being widely auto-correlated. Both bands are strongly and widely correlated with $\mathrm{GeV}$ band with $30-70$ days lag. A similar result is obtained for the radio and $\mathrm{GeV}$ correlation, where the correlation is wide with a lag of 40-70 days at the maximum of the DCF. On the other hand, the radio and optical light curves are not correlated with the $\mathrm{TeV}$ light curve, suggesting two separate mechanisms to dominate the production of $\mathrm{GeV}$ and $\mathrm{TeV}$ gamma rays.

\section{$3.3 \mathrm{GeV}$ to radio response}

Due to a strong and wide correlation of $\mathrm{GeV}$ and radio light curves, we attempted to reconstruct the radio light curve as a convolution of the $\mathrm{GeV}$ one with a response profile (see Fig. 2). The original and synthetic radio light curves are shown in Fig. 3. The response profile is defined by the equations (1) and (2) of section 3.2 of [25]. We find that the profile has $t_{\text {rise }}=3$ days, $t_{\text {fall }}=7.7$ days, $\rho(v)=1.36, \phi(v)=0.36$. An additional delay $\Delta t=43$ days was added. Such a delay was also introduced for $3 \mathrm{C} 273$ in [26]. We can reproduce the radio light curve $\left(\chi_{v}^{2}=1.2\right)$ except a fast radio flare near MJD 56897.

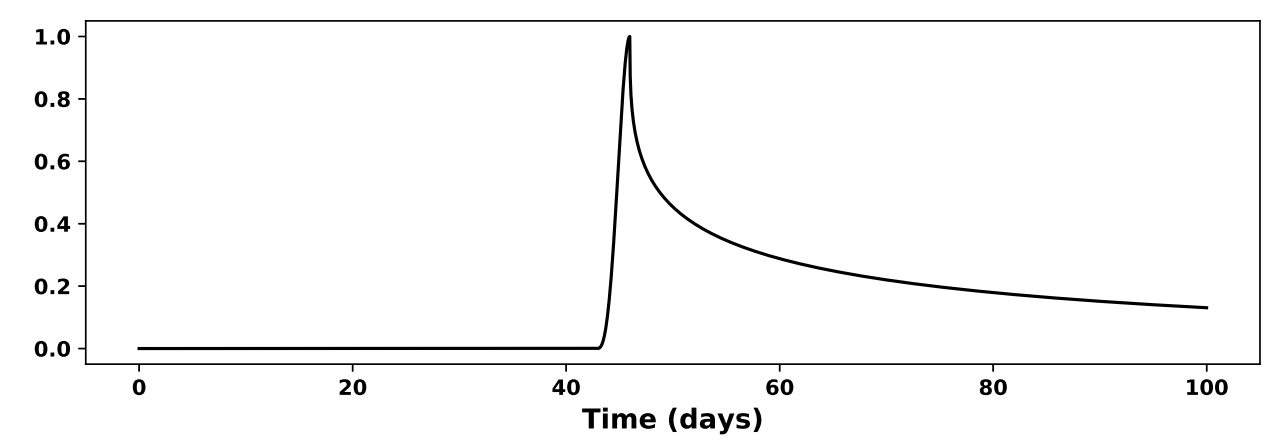

Figure 2: $\mathrm{GeV}$ to radio response profile. The $y$ axis is in arbitrary units. 


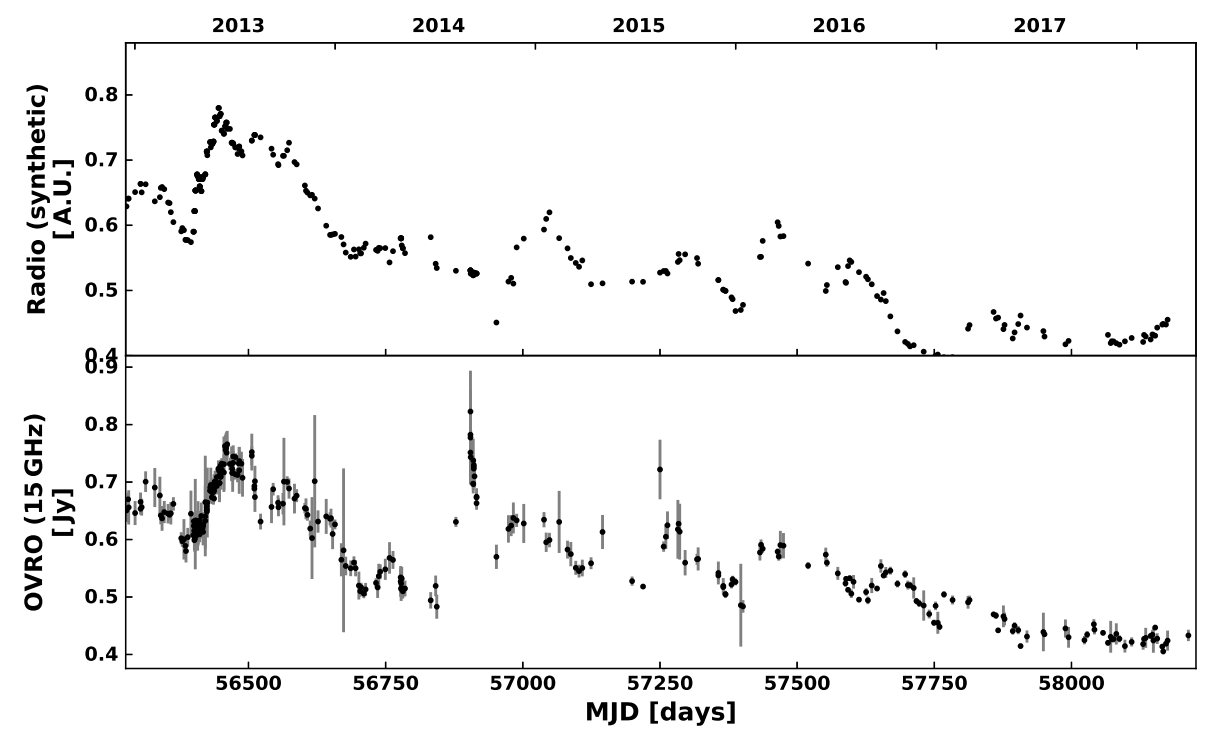

Figure 3: Synthetic radio light curve (top) derived from Fermi LAT light curve and OVRO $15 \mathrm{GHz}$ radio light curve (bottom).

\section{Results and conclusions}

The analysis of 5.5 years long multi-wavelength light curves of Mrk 421 gives an interesting insight into emission processes of the blazar. We report two main results:

1. Mrk 421 has the highest variability in X-rays and in the TeV band. $95 \%$ of the short X-ray and $\mathrm{TeV}$ flares are coincident. The $\mathrm{TeV}$ light curve is strongly correlated with $\mathrm{X}$-rays with no significant lag $((0.26 \pm 0.46)$ days $(1 \sigma))$.

2. The radio light curve can be reproduced by convolving the GeV light curve with a fast rise and a slow decay response profile with a delay of $\sim 43$ days.

The fractional variability of Mrk 421 and the correlated $\mathrm{TeV}$ and X-ray emission indicate that the main source of variability is dominated by a synchronous change of the cutoff energies of the low and high-energy components.

The radio emission variability can be reproduced by a delayed response of the $\mathrm{GeV}$ variability. This is a strong indication that synchrotron processes dominate the low energy emission component. The radio response, indicating a fast rise after a delay of $\approx 43$ days and a slow decay over $\sim 150$ days can be interpreted as an emitting region moving outwards and becoming first transparent to gamma rays and later to the radio emission from the radio core. The fast rise after a delay may indicate either a discontinuity in the jet direction, in particles density or in magnetic field.

The excellent correlation at zero lag between the $\mathrm{TeV}$ and X-ray light curves of Mrk 421 indicates that these two emissions are driven by the same physical parameter and are consistent with the leptonic emission scenario. This can be driven by variations of the electron maximal energy, or by e.g. the magnetic field that would affect both electrons and protons. The variability of Mrk 421 is therefore controlled by two independent parameters, the amplitude and the cut-off 
energy. On the other hand, the observed variability in the X-rays for protons, according to the hadronic emission scenario, does not match the observations. The proton acceleration times for lepto-hadronic models are also much longer than the time delay between $\mathrm{TeV}$ and $\mathrm{X}$-ray light curves yielding that proton-synchrotron emission cannot be responsible for the $\mathrm{TeV}$ band.

Acknowledgements. This research has made use of public data from the OVRO 40-m telescope [27], the Bok Telescope on Kitt Peak and the 1.54 m Kuiper Telescope on Mt. Bigelow [18], MAXI [16], Fermi LAT [18] and Swift [28].

\section{References}

[1] A. A. Abdo, M. Ackermann, M. Ajello, L. Baldini, J. Ballet, G. Barbiellini et al., Fermi Large Area Telescope Observations of Markarian 421: The Missing Piece of its Spectral Energy Distribution, 736 (2011) 131 [1106.1348].

[2] M. Cerruti, A. Zech, C. Boisson and S. Inoue, A hadronic origin for ultra-high-frequency-peaked BL Lac objects, 448 (2015) 910 [1411 . 5968].

[3] A. Mücke and R. J. Protheroe, A proton synchrotron blazar model for flaring in Markarian 501, Astroparticle Physics 15 (2001) 121 [astro-ph/0004052].

[4] J. Aleksić, L. A. Antonelli, P. Antoranz, A. Babic, U. Barres de Almeida, J. A. Barrio et al., Rapid and multiband variability of the TeV bright active nucleus of the galaxy IC 310, 563 (2014) A91 [1305 . 5147].

[5] C. Tanihata, J. Kataoka, T. Takahashi and G. M. Madejski, Evolution of the Synchrotron Spectrum in Markarian 421 during the 1998 Campaign, 601 (2004) 759 [astro-ph/0310592].

[6] J. Aleksić, S. Ansoldi, L. A. Antonelli, P. Antoranz, A. Babic, P. Bangale et al., The 2009 multiwavelength campaign on Mrk 421: Variability and correlation studies, 576 (2015) A126 [1502 . 02650].

[7] D. Dorner, A. Biland, T. Bretz, J. Buss, S. Einecke, D. Eisenacher et al., FACT - Long-term Monitoring of Bright TeV-Blazars, arXiv e-prints (2013) [1311.0478].

[8] H. Anderhub, M. Backes, A. Biland, V. Boccone, I. Braun, T. Bretz et al., Design and operation of FACT - the first G-APD Cherenkov telescope, Journal of Instrumentation 8 (2013) P06008 [1304 . 1710].

[9] D. Hildebrand, M. L. Ahnen, M. Balbo, A. Biland, T. Bretz, J. Buss et al., Using Charged Cosmic Ray Particles to Monitor the Data Quality of FACT, 35th International Cosmic Ray Conference, Proceedings of Science 301 (2017) 779.

[10] M. Mahlke, T. Bretz, J. Adam, L. M. Ahnen, D. Baack, M. Balbo et al., FACT - Searching for periodicity in five-year light-curves of Active Galactic Nuclei, 35th International Cosmic Ray Conference, Proceedings of Science 301 (2017) 612.

[11] T. Bretz, Zenith angle dependence of the cosmic ray rate as measured with imaging air-Cherenkov telescopes, Astroparticle Physics 111 (2019) 72 [1902 . 03875].

[12] B. Riegel, T. Bretz, D. Dorner, K. Berger and D. Höhne, A systematic study of the interdependence of IACT image parameters, 29th International Cosmic Ray Conference $\mathbf{5}$ (2005) 215. 
[13] W. B. Atwood, A. A. Abdo, M. Ackermann, W. Althouse, B. Anderson, M. Axelsson et al., The Large Area Telescope on the Fermi Gamma-Ray Space Telescope Mission, 697 (2009) 1071 [0902 . 1089].

[14] W. H. Baumgartner, J. Tueller, C. B. Markwardt, G. K. Skinner, S. Barthelmy, R. F. Mushotzky et al., The 70 Month Swift-BAT All-sky Hard X-Ray Survey, 207 (2013) 19 [1212.3336].

[15] D. N. Burrows, J. E. Hill, J. A. Nousek, J. A. Kennea, A. Wells, J. P. Osborne et al., The Swift X-Ray Telescope, 120 (2005) 165 [astro-ph/0508071].

[16] M. Matsuoka, K. Kawasaki, S. Ueno, H. Tomida, M. Kohama, M. Suzuki et al., The MAXI Mission on the ISS: Science and Instruments for Monitoring All-Sky X-Ray Images, 61 (2009) 999 [0906.0631].

[17] P. W. A. Roming, T. E. Kennedy, K. O. Mason, J. A. Nousek, L. Ahr, R. E. Bingham et al., The Swift Ultra-Violet/Optical Telescope, 120 (2005) 95 [astro-ph/0507413].

[18] P. S. Smith, E. Montiel, S. Rightley, J. Turner, G. D. Schmidt and B. T. Jannuzi, Coordinated Fermi/Optical Monitoring of Blazars and the Great 2009 September Gamma-ray Flare of $3 \mathrm{C}$ 454.3, arXiv e-prints (2009) [0912 . 3621].

[19] S. Vaughan, R. Edelson, R. S. Warwick and P. Uttley, On characterizing the variability properties of X-ray light curves from active galaxies, 345 (2003) 1271 [astro-ph/0307420].

[20] J. Poutanen, A. A. Zdziarski and A. Ibragimov, Superorbital variability of X-ray and radio emission of Cyg X-1 - II. Dependence of the orbital modulation and spectral hardness on the superorbital phase, 389 (2008) 1427 [0802 . 1391].

[21] J. Aleksić, S. Ansoldi, L. A. Antonelli, P. Antoranz, A. Babic, P. Bangale et al., Unprecedented study of the broadband emission of Mrk 421 during flaring activity in March 2010, 578 (2015) A22 [1412 . 3576].

[22] B. M. Peterson, I. Wanders, K. Horne, S. Collier, T. Alexander, S. Kaspi et al., On Uncertainties in Cross-Correlation Lags and the Reality of Wavelength-dependent Continuum Lags in Active Galactic Nuclei, 110 (1998) 660 [astro-ph/9802103].

[23] M. L. Ahnen, S. Ansoldi, L. A. Antonelli, P. Antoranz, A. Babic, B. Banerjee et al., Long-term multi-wavelength variability and correlation study of Markarian 421 from 2007 to 2009, 593 (2016) A91 [1605.09017].

[24] J. D. Scargle, J. P. Norris, B. Jackson and J. Chiang, Studies in Astronomical Time Series Analysis. VI. Bayesian Block Representations, 764 (2013) 167 [1207. 5578].

[25] M. Türler, T. J.-L. Courvoisier and S. Paltani, Modelling the submillimetre-to-radio flaring behaviour of 3C 273, 349 (1999) 45 [astro-ph/9906274].

[26] V. Esposito, R. Walter, P. Jean, A. Tramacere, M. Türler, A. Lähteenmäki et al., The high energy spectrum of 3C 273, 576 (2015) A122 [1503.02980].

[27] J. L. Richards, W. Max-Moerbeck, V. Pavlidou, O. G. King, T. J. Pearson, A. C. S. Readhead et al., Blazars in the Fermi Era: The OVRO $40 \mathrm{~m}$ Telescope Monitoring Program, 194 (2011) 29 [1011.3111].

[28] N. Gehrels and Swift Team, The Swift $\gamma$-ray burst mission, New Astronomy Reviews 48 (2004) 431. 